\title{
Ponds of landuse in Yangmei District and Pingzhen District, Taoyuan City, Taiwan
}

\author{
Tzu-Hui Lin ${ }^{\text {a, } *}$ \\ ${ }^{a}$ Chinese Culture University, Graduate School of Earth Science, B.A Student, chloelin0325@ gmail.com \\ * Corresponding author
}

Keywords: overlay, geographic information system, settlement, paddy field

\begin{abstract}
:
The purpose of this study is to explore the land use of the pond in Taoyuan where located in north Taiwan. Apply ponds to gather water to irrigate the paddy field is a special landscape in this area. In this study, six maps from 1921 to 2015 were selected to digitize the change of the landuse, and intersection, erase analysis functions of GIS were used to show the growth and decline process of ponds, combined with literature analysis to discuss the background of the landuse development. The results reveal that from 1921 to $1989,91 \%$ ponds were converted to paddy field. In 1989 to 1993 , only $68 \%$ paddy fields and 30\% settlements exist. The reasons for the conversion of ponds to paddy field were the construction of Shimen Reservoir in that time led to decline people's willingness to use the pond to irrigate; the reason for the increase in settlements was the increase in population. This study proved that the growth and decline of pond was absolutely related to human interaction, and has an absolute impact on agricultural use in all days.
\end{abstract}

